\title{
Resonant photon tunneling enhancement of the radiative heat transfer
}

\author{
A. I. Volokitin ${ }^{1,2}$ and B. N. J. Persson ${ }^{1}$ \\ ${ }^{1}$ Institut für Festkörperforschung, Forschungszentrum Jülich, D-52425 Jülich, Germany \\ ${ }^{2}$ Samara State Technical University, 443100 Samara, Russia
}

(Received 16 June 2003; published 28 January 2004)

\begin{abstract}
We study the dependence of the heat transfer between two semi-infinite solids on the dielectric properties of the bodies. We show that the heat transfer at short separation between the solids may increase by many orders of magnitude when the surfaces are covered by adsorbates, or can support low-frequency surface plasmons. In this case the heat transfer is determined by resonant photon tunneling between adsorbate vibrational modes, or surface plasmon modes. Using the nonlocal optic dielectric approach we study the dependence of the heat flux between two metal surfaces on the electron concentration, and compare the predictions of the local optic approximation.
\end{abstract}

DOI: $10.1103 /$ PhysRevB.69.045417

PACS number(s): $65.80 .+\mathrm{n}$

\section{INTRODUCTION}

It is well known that the radiative heat transfer between two bodies separated by $d \gg d_{T}=c \hbar / k_{B} T$ is given by the Stefan-Boltzmann law:

$$
S=\frac{\pi^{2} k_{B}^{4}}{60 \hbar^{3} c^{2}}\left(T_{1}^{4}-T_{2}^{4}\right)
$$

where $T_{1}$ and $T_{2}$ are the temperatures of solids 1 and 2, respectively. In this limiting case the heat transfer is determined by the propagating electromagnetic waves radiated by the bodies, and does not depend on the separation $d$. For $d$ $<d_{T}$ the heat transfer increases by many orders of magnitude due to the evanescent electromagnetic waves that decay exponentially into the vacuum; this is often referred to as photon tunneling. At present there is an increasing number of investigations of heat transfer due to evanescent waves in connection with the scanning tunneling microscopy and the scanning thermal microscopy (STM) under ultrahigh vacuum conditions. ${ }^{1-7}$ It is now possible to measure extremely small amounts of heat transfer into small volumes. ${ }^{8}$ STM can be used for local heating of the surface, resulting in local desorption or decomposition of molecular species, and this offers further possibilities for the STM to control the local chemistry on a surface.

The efficiency of the radiative heat transfer depends strongly on the dielectric properties of the media. In Refs. 3,5 and 6 and it was shown that the heat flux can be greatly enhanced if the conductivities of the materials are chosen to maximize the heat flow due to photon tunneling. At room temperature the heat flow is maximal at conductivities corresponding to semimetals. In fact, only a thin film $(\sim 10 \AA)$ of a high-resistivity material is needed to maximize the heat flux. Another enhancement mechanism of the radiative heat transfer can be connected with resonant photon tunneling between states localized on different surfaces. Recently it was discovered that resonant photon tunneling between surface plasmon modes gives rise to extraordinary enhancement of the optical transmission through subwavelength hole arrays. ${ }^{9}$ The same surface mode enhancement can be expected for the radiative heat transfer (and the van der Waals friction ${ }^{10}$ ) if the frequency of these modes is sufficiently low to be excited by thermal radiation. At room temperature only the modes with frequencies below $\sim 10^{13} \mathrm{~s}^{-1}$ can be excited. For normal metals surface plasmons have too much high frequencies; at thermal frequencies the dielectric function of normal metals becomes nearly purely imaginary, which exclude surface plasmon enhancement of the heat transfer for good conductors. However surface plasmons for semiconductors are characterized by much smaller frequencies and damping constants, and they can give an important contribution to the heat transfer. Recently, enhancement of the heat transfer due to resonant photon tunneling between surface plasmon modes localized on the surfaces of the semiconductors was predicted. ${ }^{7}$ The authors studied the radiative heat transfer between a small particle, considered as a pointlike dipole, and a flat surface. However, this treatment can be applied for scanning probe microscopy only when $R \ll d$, where $R$ and $d$ are the radius of the particle and the separation between the particle and the surface, respectively. In the opposite limit $R \gg d$, which is more appropriate for the scanning probe microscopy, the heat transfer between the tip and the surface can, in the first approximation, be modeled by the heat transfer between two semi-infinite solids. In this case the multiple scattering of the electromagnetic waves by the surfaces of the bodies, which was not taken into account in Ref. 7, becomes important in the photon tunneling. In particular, at sufficiently small separation $d$, the photons go back and forth several times in the vacuum gap, building up a coherent constructive interference in the forward direction much as would occur in resonant electron tunneling. In this case the surface plasmons on the isolated surfaces combine to form a "surface plasmon molecule," in much the same way as electronic states of isolated atoms combine to form molecular levels. This will result in a very weak distance dependence of the heat flux, because the photon transmission probability does not depend on $d$ in this case (see below). For large $d$ the sequential tunneling is more likely, where a surface plasmon mode decays by emitting a photon, which tunnels to the other surface where it excites a plasmon, and then couples to the other excitations in the media and exit. Other surface modes which can be excited by thermal radia- 
tion are adsorbate vibrational modes. Especially for parallel vibrations these modes may have very low frequencies.

All information about the long-range electromagnetic interaction between two noncontacting bodies is contained in the reflection factors of the electromagnetic field. At present very little is known about the reflection factors for large wave vectors and for extremely small frequencies. In our previous calculations of the radiative heat transfer and van der Waals friction ${ }^{5,6,11,12}$ we mostly considered good conductors. In this case it was shown that the important contribution comes from the nonlocal optic effects in the surface region. However it was shown that the radiative heat transfer and van der Waals friction become much larger for highresistivity material, for which the volume contribution from nonlocal effects is also important. Nonlocal optic refers to the fact that the current at point $\mathbf{r}$ depends on the electric field not only at point $\mathbf{r}$, as it is assumed in the local optic approximation, but also at points $\mathbf{r}^{\prime} \neq \mathbf{r}$ in a finite region around the point $\mathbf{r}$. In the case when both points are located outside the surface region the dielectric response function can be expressed through the dielectric function appropriate for the semi-infinite electron gas. However, if one of the points $\mathbf{r}$ or $\mathbf{r}^{\prime}$ is located in the surface region, the dielectric response function will be different from its volume value, and this gives the surface contribution from nonlocality. In order to verify the accuracy of the local optic approximation we study the dependence of the radiative heat transfer on the dielectric properties of the materials within the nonlocal dielectric approach, which was proposed some years ago for the investigation of the anomalous skin effects. ${ }^{13}$

\section{THEORY}

The problem of the radiative heat transfer between two flat surfaces had been considered some years ago by Polder and Van Hove ${ }^{1}$, Levin and Rytov ${ }^{2}$ and more recently by Pendry, ${ }^{3}$ and Volokitin and Persson. ${ }^{5,6}$ Polder and Van Hove were the first who obtained the correct formula for the heat transfer between two flat surfaces. In their investigation they used Rytov's theory ${ }^{14,15}$ of the fluctuating electromagnetic field. However, they presented their result only for identical media, and within the local optic approximation. In the subsequent treatment, they made numerical calculations not of the heat flux itself, but of its derivative with respect to temperature, i.e., their result is only for small temperature differences. Unfortunately, their paper contains no analytical formulas in closed form. Levin and Rytov ${ }^{2}$ used the generalized Kirchhoff's law ${ }^{15}$ to obtain an expression for the radiative heat transfer between two semi-infinite media in the impedance approximation. The case of good conductors was investigated in detail both in the normal and in the anomalous skin effect region. Pendry ${ }^{3}$ proposed a more compact derivation of the formula for the heat flux between two semi-infinite bodies due to evanescent waves, and calculated the heat transfer between a point dipole and a surface. Volokitin and Persson ${ }^{5}$ considered the problem of the heat transfer between two flat surfaces as a particular case of the general approach for calculation of the heat transfer. They investigated numerically the dependence of the heat flux on the dielectric prop- erties of the bodies, and found that for good conductors, even for very small distances, the heat flux is dominated by retardation effects. They also showed that the heat flux is maximal at conductivities corresponding to the semimetal.

According to Refs. 1-3, and 5 the heat transfer between two semi-infinite bodies, separated by a vacuum gap with the width $d$, is given by the formula

$$
S=\int_{0}^{\infty} d \omega\left(\Pi_{1}-\Pi_{2}\right) M,
$$

where

$$
\begin{aligned}
M= & \frac{1}{4 \pi^{2}} \int_{0}^{\omega / c} d q q \frac{\left[1-\left|R_{1 p}(\omega)\right|^{2}\right]\left[1-\left|R_{2 p}(\omega)\right|^{2}\right]}{\left|1-e^{2 i p d} R_{1 p}(\omega) R_{2 p}(\omega)\right|^{2}} \\
& +\frac{1}{\pi^{2}} \int_{\omega / c}^{\infty} d q q e^{-2 k d} \frac{\operatorname{Im} R_{1 p}(\omega) \operatorname{Im} R_{2 p}(\omega)}{\left|1-e^{-2 k d} R_{1 p}(\omega) R_{2 p}(\omega)\right|^{2}} \\
& +[p \rightarrow s],
\end{aligned}
$$

and the symbol $[p \rightarrow s]$ stands for the terms which can be obtained from the first two terms by replacing the reflection factor $R_{p}$ for the $p$-polarized electromagnetic waves with the reflection factor $R_{s}$ for $s$-polarized electromagnetic waves, and $p=\left[(\omega / c)^{2}-q^{2}\right]^{1 / 2}, k=|p|$. The Planck function of solid 1 is

$$
\Pi_{1}(\omega)=\hbar \omega\left(e^{\hbar \omega / k_{B} T_{1}}-1\right)^{-1},
$$

and similar for $\Pi_{2}$. The contributions to the heat transfer from the propagating $(q<\omega / c)$ and evanescent $(q>\omega / c)$ electromagnetic waves are determined by the first and the second terms in Eq. (3), respectively.

Let us first consider some general consequences of Eq. (3). In the case of heat transfer through free photons ( $q$ $\leqslant \omega / c$ ), the transfer is maximal when both bodies are perfectly black and have zero reflection coefficient, $R=R_{r}$ $+i R_{i}=0$. Now, what is the photon tunneling equivalent of a blackbody? For $q>\omega / c$ there are no constraints on the reflection coefficient $R(q, \omega)$ other than that $\operatorname{Im} R(q, \omega)$ is positive. Therefore, assuming identical surfaces, we are free to maximize the transmission coefficient corresponding to the photon tunneling:

$$
T=\frac{R_{i}^{2} e^{-2 k d}}{\left|1-e^{-2 k d} R^{2}\right|^{2}} .
$$

This function is a maximal when ${ }^{3}$

$$
R_{r}^{2}+R_{i}^{2}=e^{2 k d}
$$

so that $T=1 / 4$. Substituting this result in Eq. (3) gives the maximal evanescent contribution

$$
\left(S_{z}\right)_{\max }^{\operatorname{evan}}=\frac{k_{B}^{2} T^{2} q_{c}^{2}}{24 \hbar},
$$

where $q_{c}$ is a cutoff in $q$, determined by the properties of the material. It is clear that the largest possible $q_{c} \sim 1 / a$, where $a$ is an interatomic distance. Thus, from Eq. (7) we get the 
upper boundary for the radiative heat transfer at room temperature: $\left(S_{z}\right)_{\max } \sim 10^{12} \mathrm{~W} \mathrm{~m}^{-2}$.

Let us rewrite the denominator of the integrand in the evanescent wave term in Eq. (3) in the form

$$
\begin{aligned}
\left|1-e^{-2 k d} R^{2}\right|^{2}= & {\left[\left(1-e^{-k d} R_{r}\right)^{2}+e^{-2 k d} R_{i}^{2}\right] } \\
& \times\left[\left(1+e^{-k d} R_{r}\right)^{2}+e^{-2 k d} R_{i}^{2}\right] .
\end{aligned}
$$

The conditions for resonant photon tunneling are determined by equation

$$
R_{r}\left(\omega_{ \pm}(q)\right)= \pm e^{k d} .
$$

Since for evanescent electromagnetic waves there is no restriction on the magnitude of the real part or the modulus of $R$, Eq. (9) can be fulfilled even when $\exp (-2 k d) \ll 1$. Thus, resonant denominators can occur also for $R_{r}^{2} \gg 1$. Close to resonance we can write

$$
\begin{aligned}
& \left(1 \pm e^{-k d} R_{r}\right)^{2}+e^{-2 k d} R_{i}^{2} \\
& \quad \approx e^{-2 k d} R_{r}^{\prime 2}\left(\omega_{ \pm}\right)\left\{\left(\omega-\omega_{ \pm}\right)^{2}+\left[R_{i}\left(\omega_{ \pm}\right) / R_{r}^{\prime}\left(\omega_{ \pm}\right)\right]^{2}\right\},
\end{aligned}
$$

where

$$
R_{r}^{\prime}\left(\omega_{ \pm}\right)=\left.\frac{d R_{r}(\omega)}{d \omega}\right|_{\omega=\omega_{ \pm}},
$$

which leads to the following contribution to the heat flux:

$$
S_{ \pm} \approx \frac{1}{4 \pi} \int_{0}^{q_{c}} d k k\left[\Pi_{1}\left(\omega_{ \pm}\right)-\Pi_{2}\left(\omega_{ \pm}\right)\right] \frac{R_{i}\left(\omega_{ \pm}\right)}{R_{r}^{\prime}\left(\omega_{ \pm}\right)} .
$$

The parameter $q_{c}$ in this expression defines the region 0 $<q<q_{c}$ where the two-pole approximation is valid. To proceed further, let us make the following simplifications. Close to a pole we can use the approximation

$$
R=\frac{a}{\omega-\omega_{0}-i \eta}
$$

where $a$ is a constant. Then from the resonant condition (9) we get

$$
\omega_{ \pm}=\omega_{0} \pm a e^{-k d} .
$$

For the two-pole approximation to be valid the difference $\Delta \omega=\left|\omega_{+}-\omega_{-}\right|$must be greater than the width $\eta$ of the resonance. From this condition we get $q_{c} \leqslant \ln (2 a / \eta) / d$. For short distances the parameter $q_{c}$ defines the value of $q$ where the solution of Eq. (9) ceases to exist.

For $\omega_{0}>a$ and $q_{c} d>1$, from Eq. (11) we get

$$
S_{ \pm}=\frac{\eta q_{c}^{2}}{8 \pi}\left[\Pi_{1}\left(\omega_{0}\right)-\Pi_{2}\left(\omega_{0}\right)\right] .
$$

Note that the explicit $d$ dependence has dropped out of Eq. (13). However, $S$ may still be $d$ dependent, through the $d$ dependence of $q_{c}$. For small distances one can expect that $q_{c}$

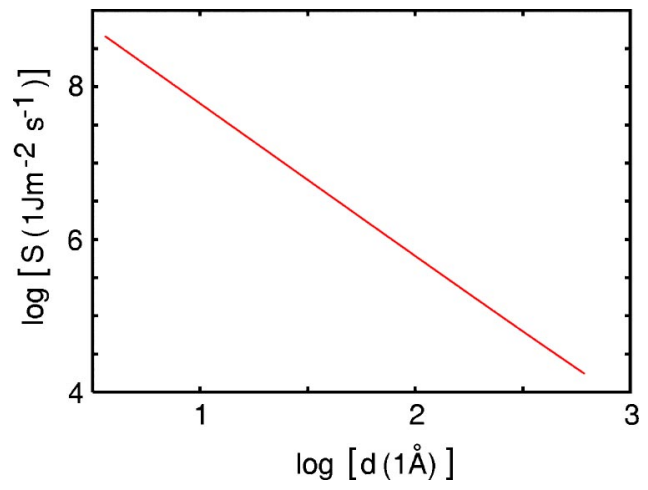

FIG. 1. The heat flux between two semiconductor surfaces as a function of separation $d$. One body is at zero temperature and the other at $T=273 \mathrm{~K}$ for parameters corresponding to a surface of silicon carbide ( $\mathrm{SiC}$ ) (see text for explanation). (The log function is with basis 10.)

is determined by the dielectric properties of the material, and thus does not depend on $d$. In this case the heat transfer will also be distance independent.

\section{NUMERICAL RESULTS}

Resonant photon tunneling enhancement of the heat transfer is possible for two semiconductor surfaces which can support low-frequency surface plasmon modes. The reflection factor $R_{p}$ for clean semiconductor surface is given by Fresnel's formula

$$
R_{p}=\frac{k-s / \epsilon}{k+s / \epsilon}
$$

where

$$
s=\sqrt{k^{2}-\left(\frac{\omega}{c}\right)^{2}(\epsilon-1)},
$$

and $\epsilon$ is the bulk dielectric function. As an example, we consider two clean surfaces of silicon carbide $(\mathrm{SiC})$. The optical properties of this material can be described using an oscillator model ${ }^{16}$

$$
\epsilon(\omega)=\epsilon_{\infty}\left(1+\frac{\omega_{L}^{2}-\omega_{T}^{2}}{\omega_{T}^{2}-\omega^{2}-i \Gamma \omega}\right)
$$

with $\epsilon_{\infty}=6.7, \omega_{L}=1.8 \times 10^{14} \mathrm{~s}^{-1}, \omega_{T}=1.49 \times 10^{14} \mathrm{~s}^{-1}$, and $\Gamma=8.9 \times 10^{11} \mathrm{~s}^{-1}$. The frequency of surface plasmons is determined by condition $\epsilon_{r}\left(\omega_{p}\right)=-1$ and from Eq. (16) we get $\omega_{p}=1.78 \times 10^{14} \mathrm{~s}^{-1}$. In Fig. 1 we plot the heat flux $S(d)$ : note that the heat flux between the two semiconductor surfaces is several orders of magnitude larger than between two clean good conductor surfaces (see Fig. 3).

Another enhancement mechanism is connected with resonant photon tunneling between adsorbate vibrational modes localized on different surfaces. As an example, let us consider ions with charge $e^{*}$ adsorbed on metal surfaces. The reflection factor $R_{p}$, which takes into account the contribution from an adsorbate layer, is given by ${ }^{17}$ 


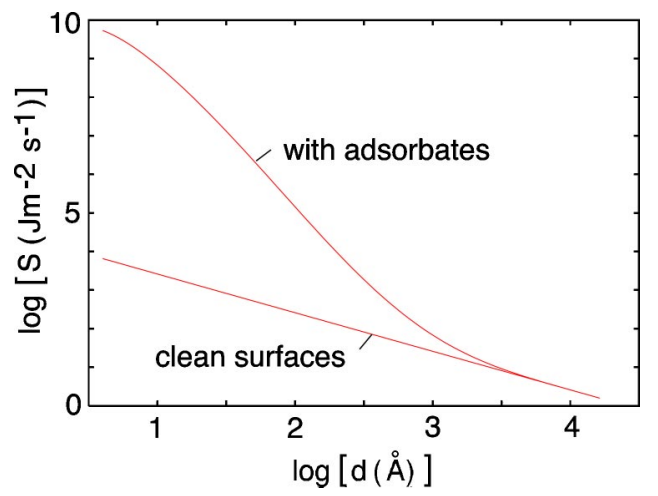

FIG. 2. The heat flux between two surfaces covered by adsorbates and between two clean surfaces, as a function of the separation $d$. One body is at zero temperature and the other at $T$ $=273 \mathrm{~K}$ for parameters corresponding to $\mathrm{K} / \mathrm{Cu}(001)$ and $\mathrm{Cu}(001)$ (Ref. 19) $\quad\left(\omega_{\perp}=1.9 \times 10^{13} \mathrm{~s}^{-1}, \quad \omega_{\|}=4.5 \times 10^{12} \mathrm{~s}^{-1}, \quad \eta_{\|}=2.8\right.$ $\times 10^{10} \mathrm{~s}^{-1}, \eta_{\perp}=1.6 \times 10^{12} \mathrm{~s}^{-1}, e^{*}=0.88 e$.) (The $\log$ function is with basis 10.)

$$
R_{p}=\frac{k-s / \epsilon+4 \pi n_{a}\left[k s \alpha_{\|} / \epsilon+q^{2} \alpha_{\perp}\right]}{k+s / \epsilon+4 \pi n_{a}\left[k s \alpha_{\|} / \epsilon-q^{2} \alpha_{\perp}\right]},
$$

and where $\alpha_{\|}$and $\alpha_{\perp}$ are the polarizabilities of adsorbates in a direction parallel and normal to the surface, respectively. $\epsilon$ is the bulk dielectric function and $n_{a}$ is the concentration of adsorbates. For clean surfaces $n_{a}=0$, and in this case formula (17) reduces to the Fresnel formula. The polarizability for an ion vibrating normal to the surface is given by

$$
\alpha_{\perp}=\frac{e^{* 2}}{M\left(\omega_{\perp}^{2}-\omega^{2}-i \omega \eta_{\perp}\right)},
$$

where $\omega_{\perp}$ is the frequency of the normal adsorbate vibration and $\eta_{\perp}$ is the damping constant. In Eq. (17) the contribution from parallel vibrations is reduced by a small factor $1 / \epsilon$. However, the contribution of parallel vibrations to the heat transfer can nevertheless be important due to the indirect interaction of the parallel adsorbate vibration with the electric field, via the metal conduction electrons. ${ }^{18}$ Thus, the small parallel component of the electric field will induce a strong electric current in the metal. The drag force between the electron flow and the adsorbates can induce adsorbate vibrations parallel to the surface. This gives the polarizability

$$
\alpha_{\|}=\frac{\epsilon-1}{4 \pi i n} \frac{e^{*}}{e} \frac{\omega \eta_{\|}}{\left(\omega_{\|}^{2}-\omega^{2}-i \omega \eta_{\|}\right)},
$$

where $n$ is the conduction electron concentration. As an illustration, in Fig. 2 we show the heat flux for the two $\mathrm{Cu}(001)$ surfaces covered by a low concentration of potassium atoms $\left(n_{a}=10^{18} \mathrm{~m}^{-2}\right)$ and the two clean $\mathrm{Cu}(001)$ surfaces. In the $q$ integral in Eq. (3) we used the cutoff $q_{c}$ $\sim \pi / a$ (where $a \approx 1 \mathrm{~nm}$ is the interadsorbate distance) because our microscopic approach is applicable only when the wavelength of the electromagnetic field is larger than the double average spacing between the adsorbates. At separation $d=1 \mathrm{~nm}$ the heat flux between two surfaces covered by

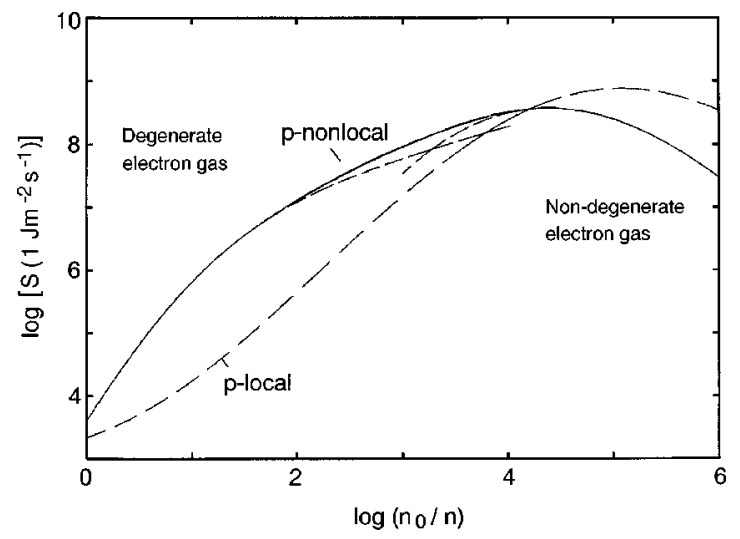

FIG. 3. The heat flux between two metal surfaces as a function of the free-electron concentration $n$. One body is at zero temperature and the other at $T=273 \mathrm{~K}$. The full line was obtained by interpolation between curves (dashed lines) calculated in the nonlocal dielectric formalism for a non-degenerate electron gas for $n$ $<n_{F} \sim 10^{25} \mathrm{~m}^{-3}$ and for a degenerate electron gas for $n>n_{F}$. Also shown are results (dashed lines) obtained within the local optic approximation. The calculation was performed with the damping constant $\tau^{-1}=2.5 \times 10^{13} \mathrm{~s}^{-1}$, separation $d=10 \AA$, and $n_{0}=8.6$ $\times 10^{28} \mathrm{~m}^{-3}$. (The $\log$ function is with basis 10 .)

adsorbates is enhanced by five orders of magnitude in comparison with the heat flux between clean surfaces, and by seven orders of magnitude in comparison with the blackbody radiation. However this enhancement of the heat flux disappears if only one of the surfaces is covered by adsorbates.

Figure 3 shows the thermal flux between two clean metal surfaces as a function of electron density $n$. In the calculations we have assumed that one body is at zero temperature and the other at $T=273 \mathrm{~K}$, and the Drude relaxation time $\tau=4 \times 10^{-14} \mathrm{~s}$. When the electron density decreases there is transition from a degenerate electron gas $\left(k_{B} T \ll \varepsilon_{F}\right.$, where $\varepsilon_{F}$ is the Fermi energy) to a nondegenerate electron gas $\left(k_{B} T \gg \varepsilon_{F}\right)$ at the density $n_{F} \sim\left(K_{B} T m\right)^{3 / 2} / \pi^{2} \hbar^{3}$, where $m$ is the electron mass. At $T=273 \mathrm{~K}$ the transition density $n_{F}$ $\sim 10^{25} \mathrm{~m}^{-3}$. The full line was obtained by interpolation between the two dashed curves, calculated in the nonlocal dielectric function formalism for the nondegenerate electron gas (valid for $n<n_{F} \approx 10^{25} \mathrm{~m}^{-3}$ ) and for the degenerate electron gas (for $n>n_{F}$ ). ${ }^{13}$ The thermal flux reaches the maximum $S_{\max } \approx 5 \times 10^{8} \mathrm{~W} \mathrm{~m}^{-2}$ at $n_{\max } \approx 10^{25} \mathrm{~m}^{-3}$, which corresponds to the dc conductivity $\sigma \approx 3 \times 10^{3}(\Omega \mathrm{m})^{-1}$. Within the local optic approximation the radiative heat transfer is maximal at $n_{L \max } \approx 10^{24} \mathrm{~m}^{-3}$ where $S_{L \max }$ $\approx 10^{9} \mathrm{~W} \mathrm{~m}^{-2}$. The thermal flux due to traveling electromagnetic waves is determined by formula (1) which gives $S_{B B}$ $=308 \mathrm{~W} \mathrm{~m}^{-2}$ for $T=273 \mathrm{~K}$.

\section{SUMMARY}

We have studied how the radiative heat transfer between two bodies depends on the dielectric properties of the media. We have found that at short distances between bodies, the thermal flux can be significantly enhanced in comparison with the blackbody radiation when the material involved can 
support low-frequency adsorbate vibrational modes, or surface plasmon modes, or the conductivity of the metals is chosen to optimize the heat transfer. This fact can be used in scanning probe microscopy for local heating and modification of surfaces.

\section{ACKNOWLEDGMENTS}

A.I.V. acknowledges financial support from DFG and B.N.J.P. acknowledges support from the European Union Smart Quasicrystals project.
${ }^{1}$ D. Polder and M. Van Hove, Phys. Rev. B 4, 3303 (1971).

${ }^{2}$ M.L. Levin, V.G. Polevoy, and S.M. Rytov, Zh. Éksp. Teor. Fiz. 79, 2087 (1980) [Sov. Phys. JETP 52, 1054 (1980)].

${ }^{3}$ J.B. Pendry, J. Phys.: Condens. Matter 11, 6621 (1999).

${ }^{4}$ A. Majumdar, Annu. Rev. Mater. Sci. 29, 505 (1999).

${ }^{5}$ A.I. Volokitin and B.N.J. Persson, Phys. Rev. B 63, 205404 (2001); Phys. Low-Dim. Struct. 5/6, 151 (2001).

${ }^{6}$ A.I. Volokitin and B.N.J. Persson, Phys. Rev. B 65, 115419 (2002).

${ }^{7}$ J.P. Mulet, K. Joulin, R. Carminati, and J.J. Greffet, Appl. Phys. Lett. 78, 2931 (2001).

${ }^{8}$ J.R. Barnes, R.J. Stephenson, M.E. Welland, Ch. Gerber, and J.K. Gimzewski, Nature (London) 372, 79 (1994).

${ }^{9}$ A. Krishnan, T. Thio, T.J. Kim, H.J. Lezec, T.W. Ebbesen, P.A. Wolf, J. Pendry, L. Martin-Moreno, and F.J. Garcia-Vidal, Opt. Commun. 200, 1 (2001).

${ }^{10}$ A.I. Volokitin and B.N.J. Persson, Phys. Rev. Lett. 91, 106101 (2003); Phys. Rev. B. 68, 155420 (2003).
${ }^{11}$ A.I. Volokitin and B.N.J. Persson, J. Phys.: Condens. Matter 11, 345 (1999); Phys. Low-Dim. Struct. 7/8, 17 (1998).

${ }^{12}$ B.N.J. Persson and A.I. Volokitin, Phys. Rev. Lett. 84, 3504 (2000).

${ }^{13}$ K.L. Kliever and R. Fuchs, Phys. Rev. 172, 607 (1968); R. Fuchs and K.L. Kliever, ibid. 186, 905 (1969).

${ }^{14}$ S.M. Rytov, Theory of Electrical Fluctuation and Thermal Radiation (Academy of Science of USSR, Moscow, 1953).

${ }^{15}$ M.L. Levin and S.M. Rytov, Theory of Equilibrium Thermal Fluctuations in Electrodynamics (Science Publishing, Moscow, 1967).

${ }^{16}$ E.D. Palik, Handbook of Optical Constants of Solids (Academic, San Diego, CA, 1985).

${ }^{17}$ D.C. Langreth, Phys. Rev. B 39, 10020 (1989).

${ }^{18}$ B.N.J. Persson and A.I. Volokitin, Surf. Sci. 310, 314 (1994).

${ }^{19}$ P. Senet, G. Witte, and J.P. Toennies, Chem. Phys. Lett. 299, 389 (1999). 